\title{
Aplicativos de acompanhamento gestacional: uma avaliação no contexto da perda gestacional
}

\author{
Sílvia Amélia Bim \\ DAINF / UTFPR \\ Curitiba - Paraná - Brasil \\ sabim@utfpr.edu.br \\ Paulo Urbano \\ C.E.S.A.R. \\ Curitiba - Paraná - Brasil \\ paulo.urbano@cesar.org.br
}

\author{
Rodrigo Pavani \\ SEDUC \\ Itacoatiara - Amazonas - Brasil \\ rodrigojapcejss@gmail.com \\ Cristiano Maciel \\ Instituto de Computação / UFMT \\ Cuiabá - Mato Grosso - Brasil \\ crismac@gmail.com
}

\begin{abstract}
RESUMO
Há diversos aplicativos para acompanhamento gestacional disponíveis no mercado. O objetivo da pesquisa relatada neste artigo é analisar como as usuárias destes aplicativos avaliam a funcionalidade de registro de uma perda gestacional. Desta forma, foram analisadas as avaliações disponíveis na Google Play de quatro aplicativos gratuitos. Os resultados indicam que o registro da perda gestacional é implementado de diferentes formas, sugerindo diferentes compreensões sobre noção de pessoa e legado digital por parte das equipes de desenvolvimento destes aplicativos. Por outro lado, as avaliações das usuárias indicam um silêncio sobre o assunto da perda gestacional.
\end{abstract}

\section{Palavras-chave}

Acompanhamento Gestacional, Legado Digital, Noção de Pessoa, Avaliação de usuários, Aplicativos

\section{Introdução}

Com o avanço de uso e capacidade dos dispositivos móveis, aplicativos são criados nas mais diversas áreas. $\mathrm{Na}$ área gestacional não poderia ser diferente. Uma busca rápida nas principais lojas virtuais de aplicativos móveis revelará um quantitativo significativo de aplicativos para acompanhamento de uma gestação, com distintas funcionalidades. Dentre estas funcionalidades está o registro da perda gestacional. A necessidade desta funcionalidade se justifica por diferentes fatores.

Permission to reproduce or distribute, in whole or in part, material extracted from this work, verbatim, adapted or remixed, as well as the creation or production from the content of such work, is granted without fee for noncommercial use, provided that the original work is properly credited. IHC 2019 - TRILHA PÔSTERES E DEMONSTRAÇÕES, Outubro 21-25, 2019, Vitória, Brasil. In Anais Estendidos do XVIII Simpósio Brasileiro sobre Fatores Humanos em Sistemas Computacionais. Porto Alegre: SBC. (C) 2019 by the author(s), in accordance with the terms of the Creative Commons Attribution-NonCommercial 4.0 International Public License (CC BY-NC 4.0).
Um destes fatores é que a perda gestacional é uma realidade. O número de óbitos fetais com menos de 22 semanas de gestação diminuiu no Brasil entre 2013 e 2016, voltando a crescer em 2017 conforme dados do Datasus: 3054 em 2013, 2991 em 2014, 2021 em 2015, 1869 em 2016 e 1981 em 2017 [4]. Entretanto, no contexto brasileiro, tanto aborto desejado quanto aborto espontâneo são tratados tabus ou como violações da regra social e por isso são evitados. Desta forma, os números podem não refletir totalmente a realidade.

Outro fator é que, segundo Maciel e Pereira [2] as aplicações necessitam considerar a vida humana em todo seu ciclo, que vai desde o nascimento até a morte. Ou seja, se uma instância de um indivíduo é criada em um software, a perspectiva de encerramento desta com a morte ou repasse dos seus bens digitais enquanto herança, devem ser considerados. Essas "instâncias" ou registro configuram aquilo que na sociologia é nomeado como representação do indivíduo ou noção de pessoa. Tal premissa deveria ser válida também para aplicativos gestacionais, todavia questiona-se nesta pesquisa se os principais aplicativos disponíveis no mercado consideram a possibilidade da perda gestacional e, caso sim, como tratam essa delicada questão?

\section{Metodologia}

Para responder à questão de pesquisa foram selecionados 4 aplicativos de acompanhamento gestacional. A seleção dos aplicativos avaliados se deu a partir da busca na Google Play ${ }^{1}$ com o termo "gravidez" e os critérios: "Apps Android", "Gratuitos" e "Quatro estrelas ou mais". A partir da análise dos cinco primeiros aplicativos apresentados e das sugestões de aplicativos semelhantes, considerou-se as notas acima de 4,5 com mais de 100.000 avaliações, com versões para Android e iOS. Com estes critérios foram selecionados: Pregnancy Week by Week ${ }^{2}$, Ovia

\footnotetext{
${ }^{2}$ https://play.google.com/store/apps/details?id=com.easymobs.pregnancy\&hl=pt-BR

\footnotetext{
${ }^{1}$ https://play.google.com/store/apps
} 
Pregnancy Tracker: Baby Due Date Countdown ${ }^{3}$, Minha gravidez e meu bebê hoje ${ }^{4}$, Gravidez+ ${ }^{5}$.

A partir da seleção dos aplicativos analisou-se as avaliações feitas por usuários(as) na Google Play sobre o tema de perda gestacional. As avaliações das(os) usuárias(os) na Google Play foram coletadas através de um algoritmo em Javascript ${ }^{6}$. Para cada conjunto de avaliações, fez-se uma busca pelas seguintes palavras: data, loss, miscarriage, abortion e stillbirth. $\mathrm{O}$ objetivo foi verificar se as(os) usuários(as) compartilhavam experiências sobre as perdas gestacionais e o modo como cada aplicativo trata deste tema.

\section{Resultados}

Para todos os aplicativos foram analisadas as avaliações em inglês. Por meio do algoritmo elaborado foram coletadas 8822 avaliações sobre o aplicativo (P) Pregnancy Week by Week (05/2016 a 06/2019); 4382 avaliações sobre o aplicativo (O) Ovia Pregnancy Tracker (06/2014 a 04/2019); 5458 avaliações sobre o aplicativo (M) Minha gravidez e meu bebê hoje (03/2011 a 06/2019); 8180 avaliações do aplicativo (G) Gravidez+ (03/2013 a 06/2019).

A palavra data foi encontrada em 120 avaliações (somando os quatro aplicativos). Entretanto, apenas 1 avaliação tinha relação com a perda gestacional. Das 27 ocorrências da palavra loss apenas 3 tinham relação com perda gestacional. A palavra miscarriage teve 23 ocorrências enquanto as palavras abortion e stillbirth tiveram 1 e 2 ocorrências respectivamente.

Embora o número de avaliações seja expressivo, raras são aquelas que explicitamente abordam o tema da perda gestacional. No caso do aplicativo Pregnancy Week by Week não foi possível identificar avaliações que abordassem o tema de perda gestacional. As avaliações do Ovia Pregnancy Tracker indicam dificuldade para encontrar a opção de registrar uma perda, reclamam com o contínuo recebimento de mensagens de uma gestação que já foi registrada como perda gestacional e, também, indicam o desejo de manter os dados de uma gestação interrompida espontaneamente. As avaliações sobre o Minha gravidez e meu bebê hoje elogiam o aplicativo pelo apoio que ele oferece em casos de aborto e perda gestacional. Por fim, as avaliações do Gravidez+ que contém a palavra data não tratam da questão do armazenamento de dados após registrar uma perda gestacional. Por outro lado, as avaliações que mencionam os termos loss e miscarriage fazem elogios sobre a forma como o aplicativo oferece apoio para estas situações.

\section{Considerações finais}

É fato que uma perda gestacional traz consigo uma série de sentimentos, em especial para os pais que aguardam o bebê. Entre esses sentimentos há o luto, que cada vez mais tem sido estudado em sistemas computacionais [3], e que tem forçado projetistas a

\footnotetext{
${ }^{3}$ https://play.google.com/store/apps/details?id=com.ovuline.pregnancy\&hl=pt-BR ${ }^{4}$ https://play.google.com/store/apps/details?id=com.babycenter.pregnancytracker\&hl $=\mathrm{pt}-\mathrm{BR}$
}

pensarem as soluções e todos seus meandros de uma forma diferenciada.

Por outro lado, a perda gestacional pode ser frustrante quando se trata de um filho desejado, talvez por isso esse tema seja tão pouco notificado nas avaliações de usuárias. Segundo [1] há para uma hipocrisia moral envolvendo o aborto. Isso significa que mesmo não notificado ou sub-representado o aborto, desejado ou espontâneo, faz parte da vida íntima, daquilo que não falamos publicamente.

O debate sobre acompanhamento gestacional em aplicativos e o registro da perda gestacional reatualiza a discussão sobre se o feto é considerado pessoa ou não. Essa pergunta opõe posições baseadas em valores sobre a vida e morte; pessoa ato e pessoa em potência.

O fato de os aplicativos não informarem de forma suficiente sobre o armazenamento de dados de uma possível perda gestacional impacta na questão do legado digital e da noção de pessoa. O feto é visto como "pessoa"? Então seu legado digital deve ser respeitado assim como de qualquer outra pessoa. As estratégias sutis para registro de uma perda gestacional representam uma noção de pessoa ou identidade que está sendo construída ou negada pelos desenvolvedores dos aplicativos.

As avaliações das usuárias indicam um silêncio sobre o assunto da perda gestacional. Entretanto, relatos significativos indicam que há outras mulheres que se sentem acolhidas pelos aplicativos e suas comunidades que tratam do tema de maneira solidária. Dentre a amostra analisada apenas uma mulher expressa que gostaria de manter os dados da gestação interrompida, revelando uma lacuna nos aplicativos.

No que ser refere ao tratamento de dados oriundos dos aplicativos, é perceptível como a análise quantitativa pode fornecer achados para questões importantes na pesquisa, orientando a análise qualitativa. Como trabalhos futuros pretende-se fazer uma avaliação por inspeção nos aplicativos e questionários e entrevistas com usuárias dos aplicativos.

\section{REFERÊNCIAS BIBLIOGRÁFICAS}

[1] Boltanski, L. (2012) As dimensões antropológicas do aborto. Revista Brasileira de Ciência Política, $\mathrm{n}^{\circ} 7$. Brasília, janeiro - abril de 2012, pp. 205-245.

[2] Maciel, C. Pereira, C.V. (2012) The influence of beliefs and death taboos in modeling the fate of digital legacy under the software developers' view. In Workshop Memento Mori: Technology design for the end of life, CHI.

[3] Maciel, C. Pereira, C.V. (2017) Technological and Human Challenges to Addressing Death in Information Systems. In: I GranDSI-BR - Grand Research Challenges in Information Systems in Brazil - 2016-2026.1 ed.: Brazilian Computer Society (SBC), p. 161-174

[4] Ministério da Saúde (BR), Datasus. Óbitos fetais. Brasília (DF); 2019. Disponível em: http://tabnet.datasus.gov.br/cgi/deftohtm.exe?sim/cnv/fet10uf.def

\footnotetext{
${ }^{5}$ https://play.google.com/store/apps/details?id=com.hp.pregnancy.lite\&hl=pt-BR

${ }^{6}$ https://github.com/paulourbano-com/pregnancy-apps-reviews
} 